\title{
Electromagnetic soil properties variability in a mine-field trial site in Cambodia
}

\author{
Ainhoa G. Gorriti ${ }^{a}$, Antonio Rañada-Shaw ${ }^{a}$, Arnold J. Schoolderman ${ }^{a}$, Jan B. Rhebergen ${ }^{a}$ \\ and Evert C. Slob. ${ }^{b}$ \\ ${ }^{a}$ TNO Defence, Security and Safety, P.O.Box 96864, 2509 JG The Hague, The Netherlands; \\ ${ }^{b}$ Department of Geotechnology, Faculty of Civil Engineering and Geosciences, Delft University \\ of Technology, Mijnbouwstraat 120, 2628 RX Delft, The Netherlands
}

\begin{abstract}
In this paper, the characterization of the electromagnetic soil properties of a blind lane used in a trial for a dual-sensor mine detector is presented. Several techniques are used and are compared here; Time Domain Reflectometry, gravimetric techniques and Frequency Domain Reflection and Transmission methods. The derived soil properties are mapped by interpolation and the resulting maps are compared with the recorded deminers' performance on the lane. Recurrent and non-predicted results from the performance of the dual-sensor detector are explained as the results of variability of certain properties.
\end{abstract}

Keywords: Soil properties, TDR, GPR, FDR, mine detection, dual-sensor mine-detector.

\section{INTRODUCTION}

For many electromagnetic (EM) geophysical applications, it is important to accurately determine the electromagnetic properties of the materials and media involved, especially useful, is the complex permittivity. The soil environment has a great impact on the usability and performance of EM mine detectors, ${ }^{1}$ i.e. metal detector or radar. In order to asses the impact of the spatial variation of the EM properties of the soil in a dual-sensor mine-detector we carried out field work on a trial site in Cambodia. Two blind lanes were set-up with minelike targets and clutter, and the performance of actual deminers was recorded. To characterize these lanes, we collected undisturbed soil samples (20 cm long) and took Time Domain Reflectometry (TDR) soil moisture measurements. Ground Penetrating Radar (GPR) profiles were also taken; these are presented in an accompanying paper. $^{2}$ The properties of undisturbed soil samples have been determined in the laboratory with a Frequency Domain Reflection and Transmission method (FDRT) ${ }^{3}$ and gravimetric techniques (GT).

In this paper, the characterization of the EM soil properties and its spatial variation derived from these measurements (FDRT and GT on undisturbed samples, and on-site TDR) is presented. The derived soil properties maps of the test lanes are compared with the observed deminer performance and, in this way, its effect is inferred.

In this case,dual-sensors mine-detectors are a combination of a metal detector (MD) and a GPR whose signal is translated into sound. They have been developed to reduce the number of false alarms that occur when using only MDs, and speed up demining operations. Metal detectors will give an alarm for every piece of metal present in a mine field while the GPR is, in principle, able to distinguish between mine like objects and clutter, thus reducing the number of false alarms. The performance of these dual-sensors mine-detectors has been tested in different trials. One of these was carried out last summer (Aug-Sep 2005) in Cambodia, where we were able to participate in a trial supporting ITEP and monitoring the operation, as well as perform measurements on two blind lanes.

The mine field was situated in a rural area, in the West of Cambodia, on the border with Thailand, see the map of Fig. 1. The succession of wars in the country has left it with millions of mines. Those found in the field were mainly PMN 2 and PMD-6 (also known as the wooden box mine), laid some 20 years ago. The NGO Mine Advisory Group (MAG) was in charge of this clearing field and their team used the metal detector Schiebel ATMID for demining. Four deminers were ascribed to the trials and were given training on the dualsensor mine-detector for two weeks. During the trials the deminers followed their usualStandard Operational

Detection and Remediation Technologies for Mines and Minelike Targets XI, edited by

J. Thomas Broach, Russell S. Harmon, John H. Holloway, Proc. of SPIE Vol. 6217,

62170T, (2006) -0277-786X/06/\$15 - doi: 10.1117/12.668974

Proc. of SPIE Vol. 6217 62170T-1 


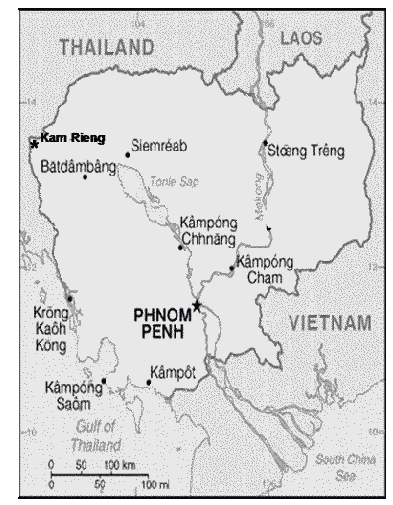

Figure 1. Kam-Rieng, location of the mine field in Cambodia.

Procedure (SOP). When a target was encountered with the metal detector, they used the dual-sensor minedetector to classify it, either as clutter or mine. The number of alarms produced by the metal detector of this dual-sensor system compared to that of the GPR was recorder and their ratio will determine the effectiveness of the dual-sensor.

Apart from the real mine field trial, blind lane was set up to monitor the performance of the dual-sensor and the deminers in a more controlled environment. While we were there, we were able to take measurements and samples of one blind lane. The lane was used in two consecutive weeks and two different target/clutter configurations were placed. A couple of days were allowed to pass before we started monitoring to avoid GPR signals due to freshly moved soil. For each groundtruth we recorded the performance of four deminers.

In this paper the electromagnetic soil properties of the lane are characterized with three different techniques, and the resulting maps are compared with the deminers performance to asses the influence of these properties in the detection performance of the dual-sensor. This is a first approach to the electromagnetic characterization of a blind lane in order to asses soil properties influence in demining activities. It raises more questions than it closes and we intend to take this as a start in the optimization of this characterization.

\subsection{Basic Theory}

The metal detector induces a time varying magnetic field by means of passing an electric current through a coil contained in its search-head. When a metallic object is present this magnetic field will induce eddy currents in this object which in response will yield a secondary magnetic field. This secondary magnetic field is detected by the search-head. The frequencies of the fields used are in the order of hundreds to thousands of $\mathrm{Hz}$, and the conductivity of the soil and the metallic target are in general very different.

The working principle of the GPR is based on the propagation of electromagnetic waves at higher frequencies, from hundreds to thousands of $\mathrm{MHz}$. At these frequencies the propagation of the waves through the subsurface is determined by the complex velocity $c^{*}$,

$$
c^{*}=\frac{c_{0}}{\sqrt{\varepsilon_{r}^{*} \cdot \mu_{r}^{*}}}
$$

where, $\varepsilon_{r}^{*}$ is the relative electrical permittivity and $\mu_{r}^{*}$ is the relative magnetic permeability. For most soils $\mu_{r}^{*}=1$ and $\varepsilon_{r}^{*}$ is determined by the permittivities of the constituents of the soils and their volume fractions. The contribution of water in the pore space dominates this property, thus, the water saturation plays a major role in the propagation of the GPR signal. The contrast between the permittivity of a target and the soil is generally not very big. The presence of other metallic objects, such as clutter, also influences the signal, but in mine fields, these objects are usually small compared to the wavelength, and are thus disregarded by the GPR.

For simplicity, from now on, we refer to the relative complex permittivity of the samples as $\varepsilon^{*}$. Note that some of the experimental techniques used only determine the real part of the permittivity, $\varepsilon$. 


\section{EXPERIMENTAL TECHNIQUE}

In this section, the different experimental techniques used are presented. They have been divided into ON-SITE and OFF-SITE measurement techniques. Some of them are devoted to the determination of the permittivity at different locations of the blind lane, for later characterization, while others record the deminers activity. The results are presented in Section 3.

\subsection{ON-SITE Measurements}

The blind lane was $1.6 \mathrm{~m}$ wide by $13 \mathrm{~m}$ long, Fig. 2. The delimited area in the figure is the actual area where the mine/clutter targets were buried, for the training and performance evaluation of the deminers.

The soil present in the mine-field was a dark peaty clay soil with weathered granite fragments no bigger than $2 \mathrm{~cm}$. Small concretions of iron oxides $(1 \mathrm{~mm})$ and charcoal fragments were present. The presence of iron oxides does not invalidate our assumption that the magnetic permeability of the soil can be assumed to be that of free space. The magnetism of iron oxides can be from different origin, depending on the material giving rise to it. Nevertheless, this magnetism never represents a measurable change in the magnetic permeability of the material at the frequencies we use. ${ }^{4}$

On the lane, the following measurements were taken:

\subsubsection{Time Domain Reflectometry}

TDR measurements were taken along and across the blind lane, see Fig. 2, in two different conditions, wet (after a storm) and dry (after a few dry days). A 2-rod probe, $15 \mathrm{~cm}$ long, was used. In this way, the average real permittivity of the first $15 \mathrm{~cm}$ of the subsurface of the lane was determined, $\varepsilon_{T D R}$. This permittivity is determined from waves traveling at frequencies higher than $300 \mathrm{MHz}$.

\subsubsection{Soil Sampling}

Undisturbed soil samples were also taken along and across the blind lane, see Fig. 2. This was done with a soil coring kit. The extracted samples varied in length from 16 up to $20 \mathrm{~cm}$. Once out of the ground they were kept in air and water tight soil sampler tubes, to avoid change in water content. Gravity measurements, see section 2.2.1, were performed to determine the real permittivity of these samples. Their complex permittivity were also determined by Frequency Domain Reflection and Transmission Measurements, see section 2.2.2.

\subsubsection{Ground Penetrating Radar}

GPR profiles were recorded along and across the lane. The GPR equipment used in this campaign was a NJJ-95A Handy Search GPR from Japan Radio Company. This particular equipment is very small, easy to transport and to use. These measurements are presented in detail in an accompanying paper "Electromagnetic soil properties variability in a minefield trial site in Cambodia and its effect on the detection of mine-like targets with a dual-sensor system".2

\subsubsection{Dual-sensor performance evaluation on the blind lane}

On the blind lane two different configurations of 12 mine-like objects and 12 clutter-like objects were buried, in two consecutive weeks. The mine-like objects were buried at different depths, from just below the surface down to $10 \mathrm{~cm}$. The deminers used the dual-sensor detector and categorized each sensor alarm as mine-like or clutter depending on the response of the dual-sensor. When only the metal detector gave an alarm and not the GPR, the alarm was categorized as clutter, and when both gave an alarm it was categorized as mine-like. For each groundtruth configuration the performance of four local deminers was recorded.

\subsection{OFF-SITE Measurements}

The undisturbed soil samples were transported from Cambodia to the Dietz Laboratorium at the Delft University of Technology. There, they were extracted from their tubes and carefully introduced into a sample holder to measure their permittivity. They were also weighed, before and after drying, and their densities were measured to determine porosity and water content. 


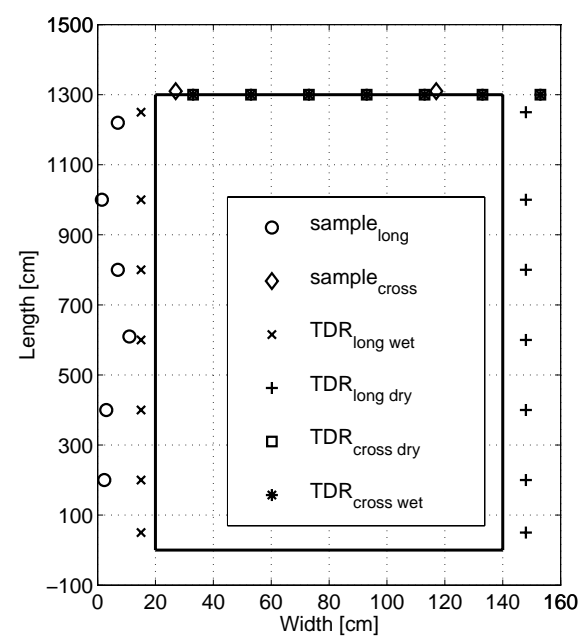

Figure 2. Blind Lane and locations at which TDR measurements and soil samples were taken. The delimited area is the actual area were the mine/clutter targets were buried. This area is $120 \mathrm{~cm}$ wide by $1300 \mathrm{~cm}$ long. Note that the locations for both $\mathrm{TDR}_{\text {crosswet }}$ and $\mathrm{TDR}_{\text {crossdry }}$ coincide.

\subsubsection{Gravimetric Measurements (GM)}

The samples' mass were measured before and after drying $\left(2-3\right.$ days in the oven at $\left.70^{\circ} \mathrm{C}\right)$ to determine their gravimetric water content $\theta_{g}$. Their density was measured with a Ultra Pycnometer and then, their porosity, $\phi$ and volumetric water content, $\theta_{v}$ were determined from

$$
\phi=1-\frac{m_{b}}{V \rho_{b}}, \quad \theta_{g}=\frac{m_{w}}{m_{b}}, \quad \theta_{v}=\theta_{g} \cdot \frac{\rho_{b}}{\rho_{w}},
$$

where $m_{b}$ is the dried bulk mass, $V$ is the total volume of the sample and $\rho_{b}$ is the bulk density, $m_{w}$ is the mass of water and $\rho_{w}$ is the density of water and taken to be $1 \mathrm{gr} / \mathrm{cm}^{3}$.

From their measured $\theta_{v}$ and using Topp ${ }^{5}$ 's formula, the real part of the permittivity was computed:

$$
\varepsilon_{G M}=3.03+9.03 \cdot \theta_{v}+146 \cdot \theta_{v}^{2}-76.7 \cdot \theta_{v}^{3},
$$

This empirical formula comes from a fitting of TDR measurements on many different soil samples with varying water contents, for frequencies between $1 \mathrm{MHz}$ and $1 \mathrm{GHz}$.

One could also use other formulas, i.e. Mixing Laws, ${ }^{6}$ to compute the permittivity from the water saturation. We have limited our experiment to the use of Topp's empirical formula because its use is widespread and well known, however, it would be desirable to study how other methods compare with the measured results. Our results suggest that this is necessary since Topp's estimate of $\varepsilon$ is very high compared to the other two techniques, see section 3.2. However, that study would be outside the scope of the present communication.

\subsubsection{Frequency Domain Reflection and Transmission Measurements}

The sample is placed in a coaxial transmission line (the sample holder is a cylinder with a $3 \mathrm{~cm}$ diameter and $10 \mathrm{~cm}$ long) and the electromagnetic reflection and transmission of the line are measured with a Network Analyzer. The full S-parameter matrix is determined from $300 \mathrm{kHz}$ to $3 \mathrm{GHz} .{ }^{3}$ From these measurements and together with a calibrated model of the line we are able to extract the relative complex permittivity, $\varepsilon^{*}$, from the electromagnetic waves propagating through the sample: 


$$
\varepsilon_{F D R T}^{*}=\left(\frac{j w}{c_{0} \gamma_{m}}\right)
$$

where $j=\sqrt{-1}, w$ is the angular frequency, $c_{0}$ is the velocity of the electromagnetic waves in vacuum and $\gamma_{m}$ is the propagation factor of the material. It is assumed that the sample is macroscopically homogeneous and isotropic. The propagation factor is directly found, determined to a high degree of accuracy, using the Propagation Matrices Method,$^{3}$ from the reflection and transmission measurements. For these samples, we used a non-golden plated brass sample holder resulting in measurements with $5 \%$ error.

The undisturbed soils samples had compacted in length due to the transport and its extraction of the original soil tube and their introduction in the sample holder. However, great care was taken in properly isolating them, so no water loss occurred. Computations show that the experienced compaction had a minor effect in the permittivity. In fact, the effect is smaller than the accuracy of the measurements and thus it was not taken into account.

Most of the samples were measured as two separated samples, one from the bottom soil, $10 \mathrm{~cm}$ long, and the other from the top soil, from 3 to $5 \mathrm{~cm}$ long. The results presented correspond to a frequency of $1 \mathrm{GHz}$, which is representative of the actual bandwith of the GPR. ${ }^{2}$

The permittivity is a frequency dependent property, and the averaged volume over which it is measured can also play an important role. For instance, if the top of the soil is wetter than the bottom, separate measurements of top and bottom will reveal different permittivities, while an averaging technique, such as the TDR will determine an average of those properties. It is, thus, important to keep this in mind when we latter compare the different results. Table 1 summarizes the different techniques used to measure the permittivity of the samples, the volume over which these properties are averaged, and the frequencies at which they are determined.

Table 1. Measured permittivities, averaged volume and frequencies used by the different measuring techniques.

\begin{tabular}{|c|c|c|c|c|}
\hline & & Length $[\mathrm{cm}]$ & Volume $\left[\mathrm{cm}^{3}\right]$ & Frequencies \\
\hline$\varepsilon_{T D R}$ & & 15 & 1200 & $>300 \mathrm{MHz}$ \\
\hline \multirow{2}{*}{$\varepsilon_{F D R T}^{*}$} & top & 5 & 23 & $1 \mathrm{GHz}$ \\
& bottom & 10 & 45 & \\
\hline \multirow{2}{*}{$\varepsilon_{G M}$} & top & 5 & 23 & $1 \mathrm{MHz}-1 \mathrm{GHz}$ \\
& bottom & 10 & 45 & \\
\hline
\end{tabular}

\section{RESULTS}

In this section we present the measured permittivities, $\varepsilon_{T D R}, \varepsilon_{F D R T}^{*}$ and $\varepsilon_{G M}$ at the different locations of the test lane, Fig. 2. These data points are used to generate interpolated permittivity maps for the blind lane, section 3.4. We also present the deminers performance for two different groundtruths ( section 3.5) and compare these to the interpolated permittivity maps (section 3.6).

\subsection{TDR Results}

Fig. 3 shows $\varepsilon_{T D R}$ for locations (see Fig. 2) along (left) and across (right) the blind lane. According to the manufacturer, these data have a $6 \%$ error. The data are separated into data taken under wet and dry conditions. From the figure, and as expected, it is clear that the permittivity corresponding to wetter conditions is higher than that of drier conditions. Along the line (left figure) the permittivity shows an alternating increasing/decreasing/increasing pattern, with a minimum at the $6 \mathrm{~m}$ location. Across the line (right figure) the permittivity follows a decreasing/increasing pattern. These data is used to generate two permittivity maps (wet and dry) of the lane by simple linear interpolation. These maps are presented in section 3.4. 

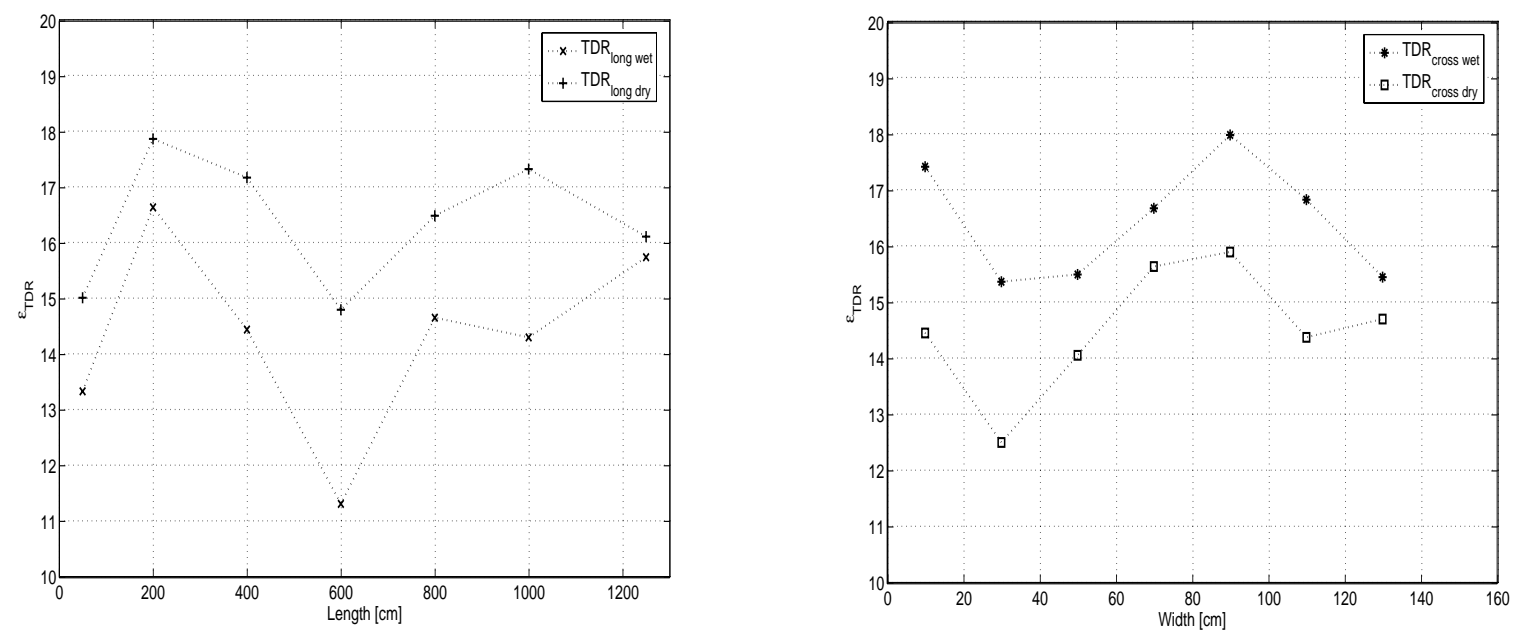

Figure 3. $\varepsilon_{T D R}$ for locations (see Fig. 2) along (left) and across (right) the blind lane.

\subsection{GM Results}

As explained in section 2.2.1, the density of the samples extracted from the lane was measured $\left(\rho_{t o p}=2.8 \mathrm{gr} / \mathrm{cm}^{3}\right.$ and $\rho_{\text {bottom }}=3.0 \mathrm{gr} / \mathrm{cm}^{3}$ ). Then, their porosity and volumetric water content were determined. From these, their $\varepsilon$ was computed with Topp's formula. These three properties are presented in Fig. 4. It should be noted that the samples corresponding to locations 6 and $10 \mathrm{~m}$ were not long enough to be separated into top and bottom samples, and therefore, they correspond to the first $10 \mathrm{~cm}$ of the subsurface. The samples presented at locations 13 and $14 \mathrm{~m}$, correspond to those taken from the cross profile of the lane, locations A and C of Fig. 2. Another sample had been extracted between these two locations, but unfortunately, it was unmeasurable.

The separation of the samples into top and bottom samples was performed because their length allowed it, but also, because it was clear that the first $5 \mathrm{~cm}$ of the samples, those closer to the surface, were different than the rest of the sample. This is because they correspond to the weathered layer. No general trend is observed in the porosity of these samples, top part of Fig. 4, however, the bottom samples have a higher water content than the top samples (middle part of Fig. 4), as one might expect. This is not the case for the samples from the cross locations.

The computed permittivities, $\varepsilon_{G M}$, from this water content using Topp's formula are very big. Usually, fully saturated soils, even very porous ones, do not exceed a permittivity of 30 . Furthermore, when these values are compared to the ones obtained with the other two techniques, their inadequacy is highlighted. We think this is because the water contents are bigger than those used by Topp to find his empirical formula and therefore another formula should be used. We will, therefore, not use these data to produce interpolated maps of the permittivity of the lane. However, as mentioned before, other formulas should be investigated.

\subsection{FDRT Results}

The FDRT technique calculates the permittivity within a margin of $5 \%$ error when the sample holder is used over its complete length. In fact, shorter samples present resonances that can mask the results. ${ }^{7}$ The top samples varied in length, but none was longer than $5 \mathrm{~cm}$, which introduced extra uncertainty. 

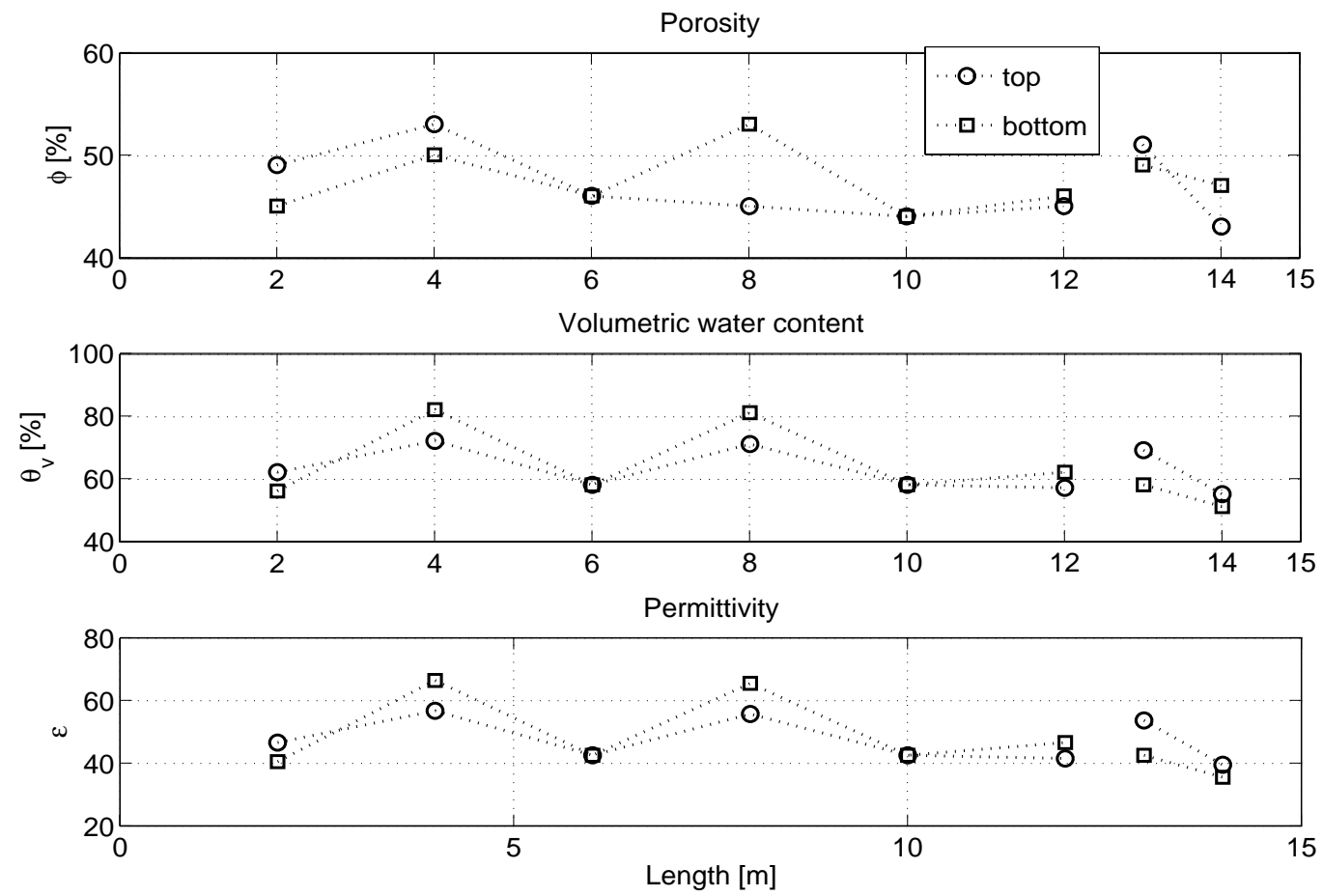

Figure 4. Porosity, volumetric water content and $\varepsilon_{G M}$ for the samples extracted from the blind lane. The samples corresponding to location 6 and 10 meters were short and could not be separated into top and bottom samples, therefore, their top and bottom values are equal. The values placed in location 13 and $14 \mathrm{~m}$, correspond to the locations $\mathrm{A}$ and $\mathrm{C}$ across the lane, see Fig 2.
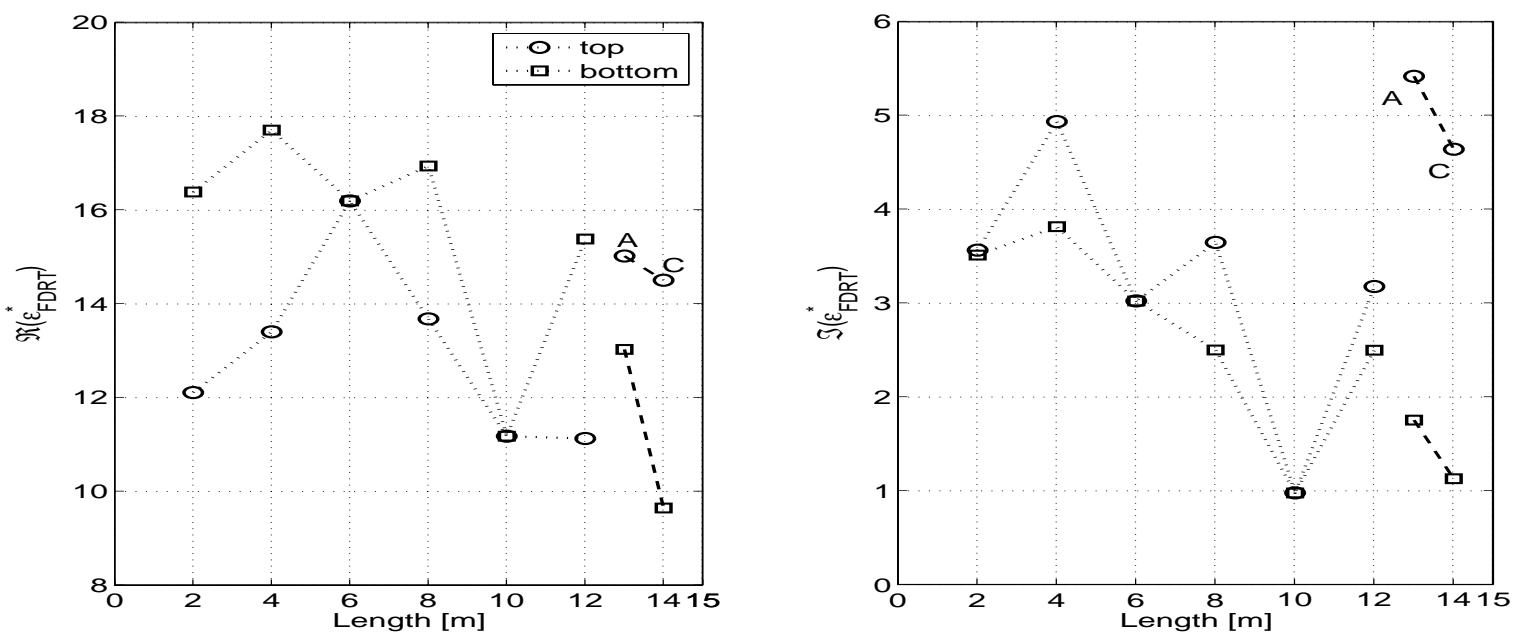

Figure 5. $\varepsilon_{F D R T}^{*}$ for the samples extracted from the blind lane. Real part on the left figure and Imaginary part on the right. As in Fig. 4, the values for top and bottom are equal, and those placed in location 13 and $14 \mathrm{~m}$, correspond to the locations $\mathrm{A}$ and $\mathrm{C}$ across the lane, see Fig 2. 
The real and imaginary parts of the permittivity of the top and bottom samples are presented in Fig. 5 . Both real and imaginary parts of the top samples from the cross profile (samples A and C) are larger than their bottom counterparts, as their higher water content suggested (see Fig. 4). For the samples from locations along the lane, the water content of the bottom samples is higher than that of the top samples (see Fig. 4) and so are the computed real parts of $\varepsilon_{F D R T}^{*}$. However, one would expect the imaginary parts also to be larger and this is not the case. We can only explain this from the extra uncertainty introduced by the fact that they were shorter than the sample holder.

Note that although $\varepsilon_{T D R}$ and $\varepsilon_{F D R T}$ measure over different volumes their values are within the same range. They also use different frequencies, but from the frequency dependent measurements of $\varepsilon_{F D R T}$ we could see that in the $200 \mathrm{MHz}$ to $2 \mathrm{GHz}$ region they were fairly constant. We can then conclude that the different volume averaging does not play a major role.

\subsection{Permittivity maps}

In this section, the generated permittivity maps are presented. We only show maps generated from $\varepsilon_{T D R}$ and $\varepsilon_{F D R T}$. They have been created by means of linear interpolation of the measured data along and across the lane. The lane has been divided into rectangles $[20 \mathrm{~cm} \times 200 \mathrm{~cm}]$ and each rectangle is assumed to have piecewise homogeneous permittivity. Across the lane, only two FDRT samples were measurable and therefore, the section area assumed to have the same permittivity is larger. Light areas correspond to drier areas (low permittivity), while dark areas correspond to wetter areas (high permittivity).

Fig. 6 shows the generated maps from $\varepsilon_{T D R}$. The variability of permittivity is higher in the wet map than in the dry. In the wet case, there is a dry band $(40$ to $60 \mathrm{~cm})$ that stretches along the lane, there is also a very wet central area $(60 \mathrm{~cm}$ wide, 60 to $120 \mathrm{~cm}$ ) expanding in the same direction. In the dry case, the contrasts are not that marked, however, the central wet area is still visible, and two drier bands stretch at its sides.

Fig. 7 shows the generated maps from $\varepsilon_{F D R T}$. The variability of permittivity is higher in the top map than in the bottom. In contrast to the TDR maps, here, the properties seem to correlate in the cross direction rather than in the long one. For the top soil there are two relatively drier areas, at the top and the bottom of the lane ( 0 to $3 \mathrm{~m}$, and 9 to $13 \mathrm{~m}$ ), that gradually dry up in the cross direction. In the center of the lane, there is a very wet band $(5$ to $7 \mathrm{~m})$ surrounded by relatively drier bands $(2$ to $5 \mathrm{~m}$ and 7 to $9 \mathrm{~m})$. The bottom map of the lane shows it is wetter than the top. A drier band is still visible at the top of the lane (9 to $11 \mathrm{~m}$ ). The central wet band that appeared in the top map is inverted in the bottom map (drier band ( 5 to $7 \mathrm{~m}$ ) adjacent to two wetter bands ( 2 to $5 \mathrm{~m}$ and 7 to $9 \mathrm{~m}$ ).

\subsection{Dual-sensor performance results}

For each groundtruth configuration the performance of four local deminers was recorded. For the sake of simplicity we will not include all results but only comment on the most relevant. As relevant we understand those results that fall under non-predicted performance and that are recurrent.

On average, the dual-sensor equipment reduced the false alarms by $30 \%$. That is, out of 10 alarms given by the metal detector only 7 were also categorized as mine-like objects by the GPR and 3 were disregarded as clutter. The dual-sensor has then the potential of speeding up real demining operations.

On wet conditions some of the deminers missed one or two deep $(10 \mathrm{~cm})$ mine-like objects with the GPR. Other mine-like objects were classified as two distinct targets under wet and dry conditions. We have assumed a detection halo of $10 \mathrm{~cm}$. However, there is not enough consistency to be able to draw any conclusions. Under dry conditions, clutter was classified as mine-like objects more often than under wet conditions. The recurrence of classifying them as two distinct targets was higher in dry conditions. These doubled classified, by 3 or more deminers, targets are shown in the left figure of Fig. 8.

It was surprising to note the recurrence of false alarms at specific location or areas. No targets were buried there, however, most or all deminers classified them coincidentally as mine-like objects or clutter. We named these non-existing targets as ghosts. These ghosts are presented in the right figure of Fig. 8.

In the next subsection these maps are compared with the ones generated from the measured permittivities.

From this comparison we can see if the soils properties variability can explain the non-predicted and recurrent performance of the dual-sensor. 

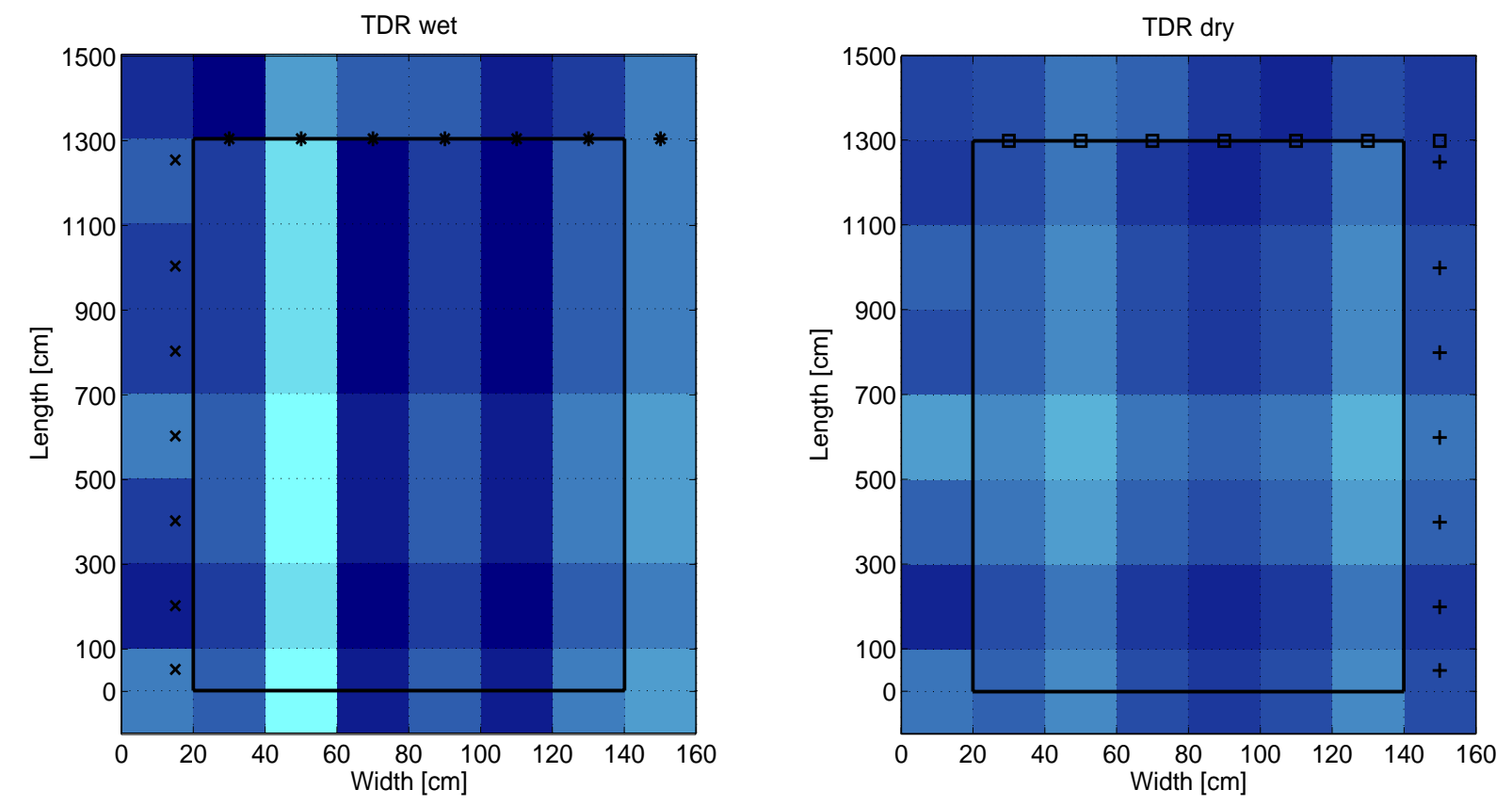

Figure 6. Interpolated maps from $\varepsilon_{T D R}$. The left figure corresponds to wet conditions and the right one to dry. The points on the figures represent the locations at which the measurements were performed.
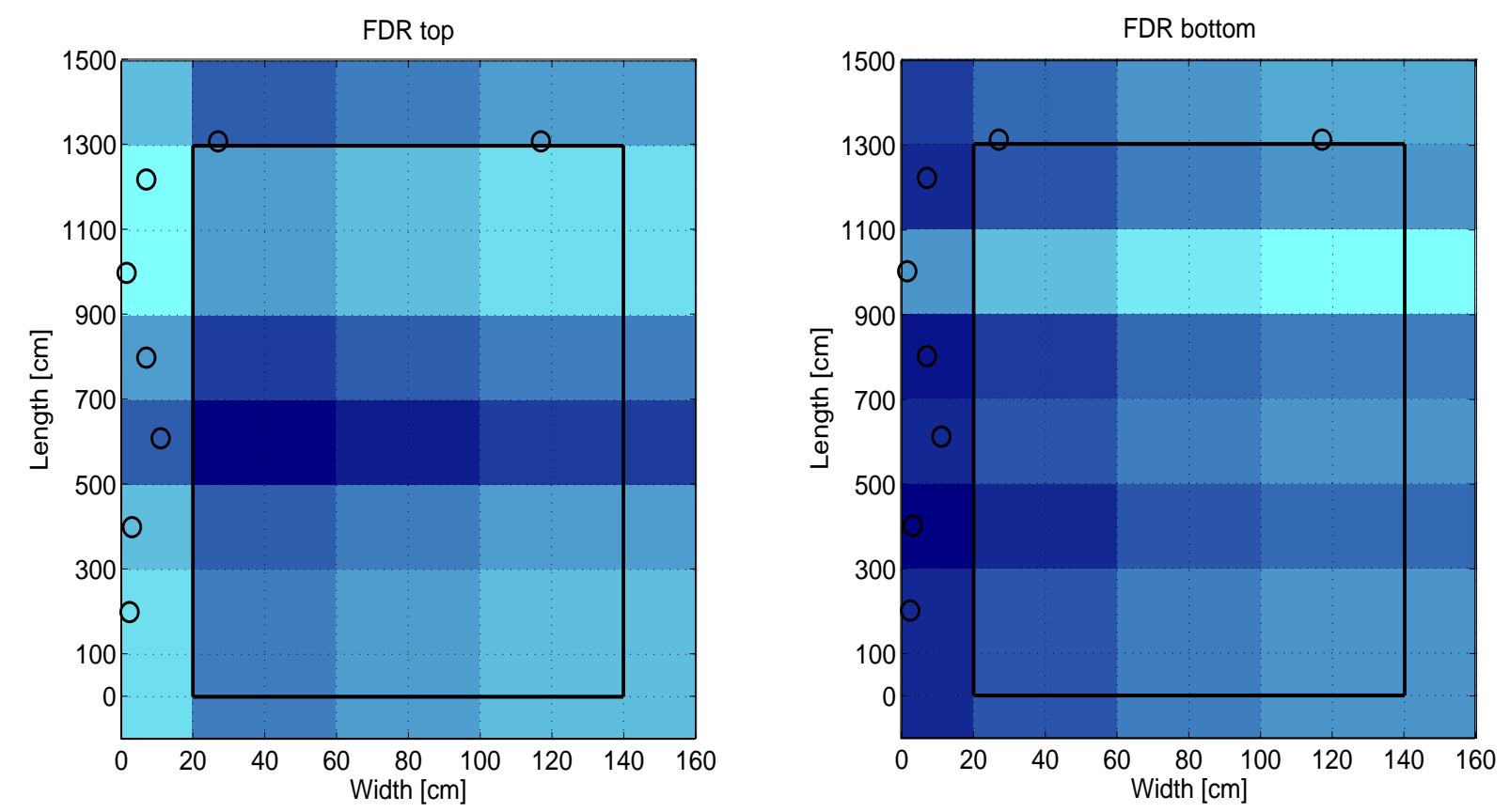

Figure 7. Interpolated maps from $\varepsilon_{F D R T}$. The left figure corresponds to the top soil $(5 \mathrm{~cm})$ and the right one to the bottom $(10 \mathrm{~cm})$. The circles on the figure represent the locations from where the samples were extracted. 

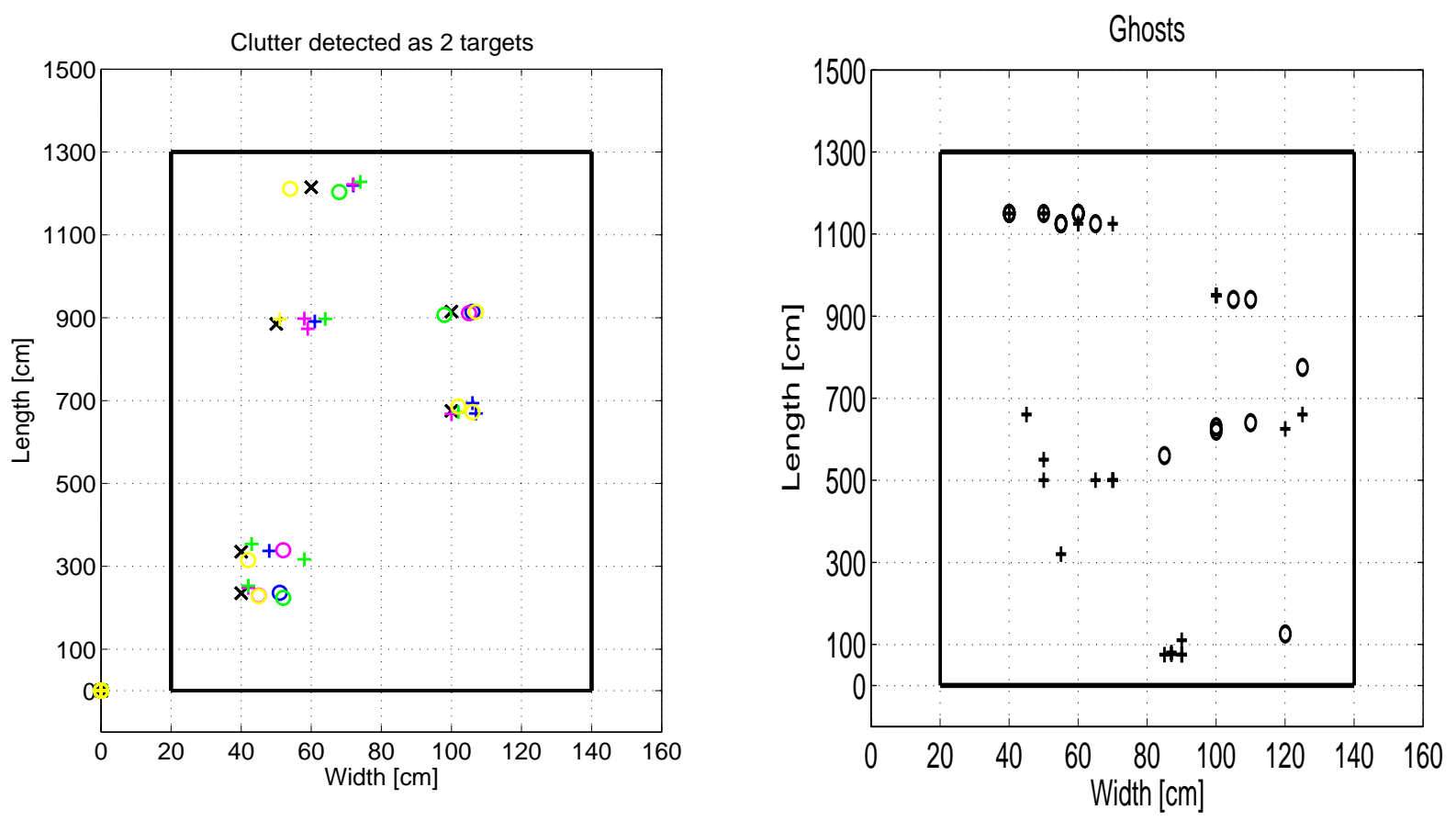

Figure 8. Left: Clutter targets (x) that were classified as 2 targets by 3 or more deminers. Circles stand for mine-like classification and crosses for clutter. Right: Ghosts targets found on the blind lane by 3 or more deminers.

\subsection{Comparison of permittivity maps and performance peculiarities of the dual-sensor}

Comparing the right map of Fig. 8 with the right map of Fig. 6, that is, the occurrence of clutter targets that were multi classified by the deminers during dry days, with the TDR map generated for dry conditions, it can be seen that most of this specific multi classified targets happen to lie in regions where the soil properties experience a change. This could explain the multi classification.

We think that the occurrence of ghosts, right part of Fig. 8, has two different explanations. When they expand shortly in the cross direction (i.e. top and bottom of left part of Fig. 8) that can be due to buried roots and/or the previous presence of mine-like objects, that were excavated. However, there is a clear band full of ghosts $(5$ to $7 \mathrm{~m}$ ) that expands for the whole width of the lane, and we think this is due to the fact that it is precisely on that band where the permittivity of the lane shows an inversion wet/dry from the top to the bottom soil, Fig. 7, when it is normally the opposite, dry to wet. This can not be explained from the TDR maps because they average over the first $15 \mathrm{~cm}$ of the subsurface and do not differentiate between top and bottom.

\section{CONCLUSIONS}

Several techniques were used to characterize the electromagnetic soil properties of a blind lane set up to monitor the performance of a dual-sensor mine-detector. Time Domain Reflectometry and Frequency Domain Reflection and Transmission methods gave the best results. These were used to map the soil properties spatial variability of the lane. The resulting maps were compared with recorded local deminers performance on the lane. Recurrent and non-predicted results from the performance of the dual sensor were explained thanks to certain properties variabilities. The separation into top and bottom soil, thanks to the FDRT measurements, proved crucial to understand the occurrence of recurrent false alarms (ghosts) at a band across the whole width of the lane. An abnormal change of properties from top to bottom, wet to dry instead of dry to wet, seems to cause these false alarms. Under dry conditions, the number of multiple classifications is higher than under wet conditions, and they also relate to changes in the permittivity of the soil. 


\section{Acknowledgments}

The field work reported in this paper was executed in the framework of the International Test and Evaluation Program for Humanitarian Demining (ITEP). The authors wish to thank the Netherlands Ministry of Defence for funding. Furthermore the authors thank MAG and Savohn, Mitl, Roth and Kim Say for a pleasant collaboration.

\section{REFERENCES}

1. J. Rhebergen and J. Ralston, "Test and evaluation protocols for gpr-based mine-detection systems; a proposal," Proceedings of SPIE 5794, pp. 941-952, 2005.

2. A. Rañada Shaw, A. Gorriti, A. Schoolderman, and J. Rhebergen, "Electromagnetic soil properties variability in a minefield trial site in cambodia and its effect on the detection of mine-like targets with a dual-sensor system," SPIE Conference (OR41), 2006.

3. A. Gorriti and E. Slob, "A new tool for accurate s-parameters measurements and permittivity reconstruction," IEEE Transactions on Geoscience and Remote Sensing 43(8), pp. 1727-1735, 2005.

4. R. Dam, W. Schlager, M. Dekkers, and J. Huisman, "Iron oxides as a cause of gpr reflections," Geophysics 67(2), pp. 536-545, 2002.

5. G. Topp, "Electromagnetic determination of soil water content: Measurements in coaxial tranmission lines," Water Resources Research 16(3), pp. 574-582, 1980.

6. A. Sihvola, Electromagnetc mixing formulas and applications, The Institution of electrical Engineers, London, 1999.

7. A. Gorriti, Electric Characterization of Sands with Heterogeneous Saturation Distribution. PhD thesis, Delft University of Technology, The Netherlands, 2004. 